\title{
Development and validation of an illustrated questionnaire to evaluate disabilities of the upper limb
}

\author{
M. Suzuki, \\ S. Kurimoto, \\ T. Shinohara, \\ M. Tatebe, \\ T. Imaeda, \\ H. Hirata
}

From Nagoya University Graduate School of Medicine, Nagoya, Japan

\footnotetext{
M. Suzuki, MD, Orthopaedic Surgeon

S. Kurimoto, MD, Orthopaedic Surgeon I. Shinohara, $\mathrm{PhD}, \mathrm{MD}$, Orthopaedic Surgeon and Hand Surgeon

M. Tatebe, PhD, MD,

Orthopaedic Surgeon and Hand Surgeon

I. H. Hirata, PhD, MD, Professor Department of Hand Surgery Nagoya University Graduate School of Medicine, 65 Tsurumai-Cho, Showa-ku, Nagoya, 466-8550, Japan.

T. Imaeda, PhD, MD, Professor

Department of Foot and Nutritional Environment College of Human Life and Environment, Kinjo Gakuin University, 1723 Omori Moriyama-ku, Nagoya, 463 8521, Japan.
}

Correspondence should be sent to $\mathrm{Dr}$ M. Suzuki; e-mail: micsuzu@med.nagoya-u.ac.jp

(C)2010 British Editorial Society of Bone and Joint Surgery doi:10.1302/0301-620X.92B7. $23410 \$ 2.00$

$J$ Bone Joint Surg [Br] 2010;92-B:963-9.

Received 10 September 2009; Accepted after revision 5 March 2010

VOL. 92-B, No. 7, JULY 2010

We have developed an illustrated questionnaire, the Hand20, comprising 20 short and easyto-understand questions to assess disorders of the upper limb. We have examined the usefulness of this questionnaire by comparing reliability, validity, responsiveness and the level of missing data with those of the Disabilities of the Arm, Shoulder and Hand (DASH) questionnaire.

A series of 431 patients with disorders of the upper limb completed the Hand20 and the Japanese version of the DASH (DASH-JSSH) questionnaire. The norms for Hand20 scores were determined in another cross-sectional study.

Most patients had no difficulty in completing the Hand20 questionnaire, whereas the DASH-JSSH had a significantly higher rate of missing data. The standard score for the Hand20 was smaller than the reported norms for the DASH.

Our study showed that the Hand20 questionnaire provided validation comparable with that of the DASH-JSSH. Explanatory illustrations and short questions which were easy-tounderstand led to better rates of response and fewer missing data, even in elderly individuals with cognitive deterioration.

The assessment of outcome is essential when evaluating the results of treatment. ${ }^{1}$ Several measures for the evaluation of the function of the upper limb have been developed. Some are joint-specific ${ }^{2,3}$ and others disease-specific. ${ }^{4,5}$ However, choosing the most suitable for assessing conditions affecting the upper limb may be difficult. The Disabilities of the Arm, Shoulder and Hand (DASH) questionnaire was devised as a region-specific measure and is now used widely. It is a self-administered questionnaire comprising 30 items which evaluate symptoms and physical function using a fivelevel Likert scale. ${ }^{6}$ Its reliability, validity, and responsiveness have been evaluated in patients with disorders of all major areas of the upper limb, that is, the shoulder, elbow, wrist and hand. ${ }^{7}$ The DASH questionnaire is also recommended for evaluating patients with disorders involving multiple joints in the upper limb. ${ }^{8}$

The DASH and its short version (Quick DASH) have been translated into several languages and studies of reliability and validity have been published for the Japanese, Swedish, German, Spanish, Italian and Chinese versions. ${ }^{9-16}$ However, some items of the questionnaire do not fit into cultures outside the United States. ${ }^{15,16}$ Guidelines for the adoption of selfreported measures indicate that cross-cultural adaptation for use in non-English speaking countries is the most difficult task, since items must not only be translated linguistically, but also must be adapted culturally to maintain the content validity of the instrument. ${ }^{17}$

In addition to potential cultural concerns, the DASH questionnaire has another issue. On its website, it is recommended for assessment disorders of the upper limb in patients aged from 18 to 65 years. ${ }^{18}$ The 36-item Short-Form Health Survey (SF-36) is another self-completed health-status measure, and is regarded as suitable for use among the elderly population. However, people over 75 years of age have had difficulty in completing the SF-36. ${ }^{19}$ In addition, elderly patients may have several disorders in different regions of the upper limbs, making the analysis more complex compared with those with a single disorder.

We have developed an illustrated questionnaire for disorders of the upper limb composed of 20 short, easy-to-understand questions. In addition, we have added explanatory illustrations to 19 of the 20 questions. Our aim was that it should offer validity, reliability and responsiveness comparable with those of the DASH questionnaire. In this study these criteria were examined and compared with the DASH-JSSH, a Japanese version of the DASH. 


\begin{tabular}{|c|c|c|c|c|c|c|c|c|c|}
\hline & Questions & \multicolumn{8}{|c|}{ Mark the point the best describing your situation } \\
\hline 1 & $\begin{array}{l}\text { Wash your face } \\
\text { with both hands. }\end{array}$ & 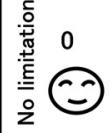 & 1 & 2 & (6) & 5 & ${ }^{6}{ }^{7}$ & 8 & 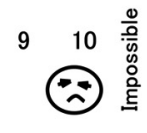 \\
\hline 2 & $\begin{array}{l}\text { Cut all } 10 \text { nails on the digits } \\
\text { of both hands properly. } \\
\text { (using a nail cutter) }\end{array}$ & 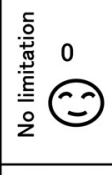 & 1 & 2 & (9) & 5 & ${ }^{6}{ }^{7}$ & 8 & $910 \frac{\frac{0}{0}}{0}$ \\
\hline 3 & $\begin{array}{l}\text { Do up shirt buttons } \\
\text { with both hands. }\end{array}$ & 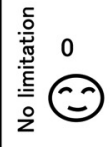 & 1 & 2 & (6) & 5 & ${ }^{6}{ }^{7}$ & 8 & $9 \overbrace{}^{10} \stackrel{\frac{0}{0}}{\frac{0}{\circ}}$ \\
\hline 4 & $\begin{array}{l}\text { Pick coins out of a purse } \\
\text { with the affected hand. }\end{array}$ & 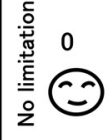 & 1 & 2 & ${ }^{3}{ }^{4}$ & 5 & ${ }^{6}{ }^{7}$ & 8 & $9 \overbrace{}^{10} \stackrel{\frac{0}{\overline{0}}}{\circ}$ \\
\hline 5 & $\begin{array}{l}\text { Turn on/off the faucet } \\
\text { with the affected hand. }\end{array}$ & 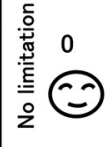 & 1 & 2 & ${ }^{3}(6)$ & 5 & ${ }^{6}{ }^{7}$ & 8 & $9 \overbrace{}^{10} \stackrel{\frac{0}{0}}{\frac{0}{0}}$ \\
\hline 6 & $\begin{array}{l}\text { Open a milk carton } \\
\text { with both hands. }\end{array}$ & 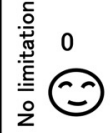 & 1 & 2 & (6) & 5 & ${ }^{6}{ }^{7}$ & 8 & $9 \overbrace{}^{10} \stackrel{\frac{\%}{0}}{\circ} \stackrel{0}{\circ}$ \\
\hline 7 & Open a PET bottle. & 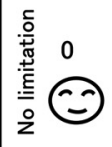 & 1 & 2 & (C) & 5 & ${ }^{6}{ }^{7}$ & 8 & 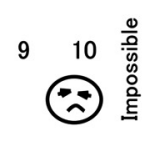 \\
\hline 8 & $\begin{array}{l}\text { Roll up and squeeze } \\
\text { a towel hard. }\end{array}$ & 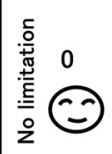 & 1 & 2 & $\overbrace{}^{3}$ & 5 & ${ }^{6}{ }^{7}$ & 8 & $9 \overbrace{}^{10} \stackrel{\frac{0}{0}}{\frac{0}{0}}$ \\
\hline 9 & $\begin{array}{l}\text { Peel an apple } \\
\text { using a knife. }\end{array}$ & 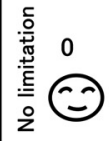 & 1 & 2 & (9) & 5 & ${ }^{6}{ }^{7}$ & 8 & $9 \overbrace{}^{10} \stackrel{\frac{0}{0}}{\frac{0}{0}}$ \\
\hline 10 & $\begin{array}{l}\text { Operate a door knob } \\
\text { and open a heavy door } \\
\text { with the affected hand. }\end{array}$ & 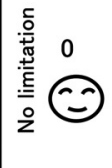 & 1 & 2 & $\overbrace{}^{3}$ & 5 & ${ }^{6}{ }^{7}$ & 0 & 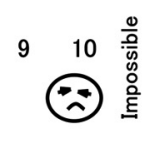 \\
\hline
\end{tabular}

Fig. 1a

Diagram showing page 1 of the English version of the Hand20 questionnaire.

\section{Patients and Methods}

The questionnaire was developed by a team of 60 people consisting of orthopaedic surgeons and occupational therapists. Initially, 300 questionnaire items were devised and subsequently reduced to 145 . Finally, 20 were extracted.
During the process of selection, we tried to choose items which appeared to reflect the function of the elbow, forearm, wrist and hand, and be the least affected by cultural differences. In order to improve understanding, we made the questions as short and as clear as possible and added 


\begin{tabular}{|c|c|c|c|c|c|c|c|c|}
\hline & Questions & & & nt the & & scribing & 15 & tion \\
\hline 11 & $\begin{array}{l}\text { Push a heavy object up } \\
\text { and onto the shelf } \\
\text { overhead using both hands. } \\
\text { (about } 5 \mathrm{~kg} \text { ) }\end{array}$ & 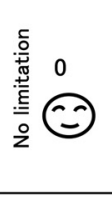 & 2 & ${ }^{3}{ }^{4}$ & 5 & ${ }^{6}$ (ii) & & 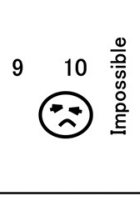 \\
\hline 12 & $\begin{array}{l}\text { Hang wet clothes on } \\
\text { a clip hanger. }\end{array}$ & 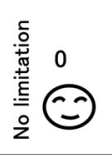 & 2 & $\left(^{3}\right)^{4}$ & 5 & (Di) & 8 & $9 \overbrace{}^{10} \frac{\frac{0}{0}}{\frac{0}{0}}$ \\
\hline 13 & $\begin{array}{l}\text { Wash your hair } \\
\text { with both hands. }\end{array}$ & 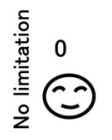 & 2 & ${ }^{3}(9)$ & 5 & (10) & & $9 \stackrel{\substack{\frac{0}{0} \\
0}}{\stackrel{0}{0}}$ \\
\hline 14 & $\begin{array}{l}\text { Turn over pages of } \\
\text { a newspaper } \\
\text { with the affected hand. }\end{array}$ & 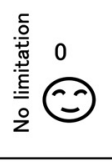 & 2 & $0^{3}(6)$ & 5 & ${ }^{6}{ }^{7}$ & 8 & 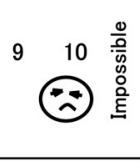 \\
\hline 5 & $\begin{array}{l}\text { Do manual work } \\
\text { without too much } \\
\text { difficulty. }\end{array}$ & 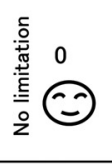 & 2 & $\left.{ }^{3}(9)\right)^{4}$ & 5 & ${ }^{6}$ (ii) & 8 & 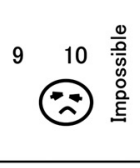 \\
\hline 16 & $\begin{array}{l}\text { Do you hesitate to show } \\
\text { people your affected } \\
\text { hand for cosmetic reasons? }\end{array}$ & 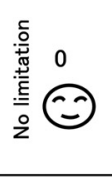 & 2 & $\left(^{3}\right)^{4}$ & 5 & ${ }^{6}{ }^{7}$ & 8 & 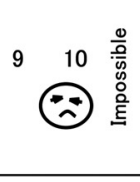 \\
\hline 17 & $\begin{array}{l}\text { Do you experience } \\
\text { difficulties in } \\
\text { recreational activities } \\
\text { (painting, knitting, sports)? }\end{array}$ & 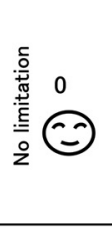 & 2 & $\left.{ }^{3}(1)\right)^{4}$ & 5 & 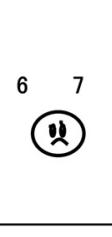 & 8 & $9 \overbrace{}^{10} \frac{\frac{0}{0}}{\overline{6}}$ \\
\hline 18 & $\begin{array}{l}\text { Do you experience } \\
\text { difficulties in } \\
\text { activities of daily living? }\end{array}$ & 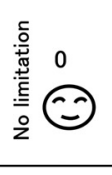 & 2 & ${ }^{3}(6)$ & 5 & ${ }^{6}$ (ii) & 8 & $9 \overbrace{}^{10} \frac{\frac{0}{0}}{\frac{0}{0}}$ \\
\hline 19 & $\begin{array}{l}\text { How much pain do you have } \\
\text { in your affected hand? }\end{array}$ & 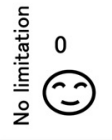 & 2 & $3^{3}{ }^{4}$ & 5 & ${ }^{6}{ }^{7}$ & 8 & $9 \overbrace{}^{10} \stackrel{\frac{0}{0}}{\frac{0}{0}}$ \\
\hline 20 & $\begin{array}{l}\text { Do you feel less } \\
\text { confident because of } \\
\text { your affected hand? }\end{array}$ & 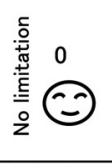 & 2 & 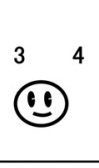 & 5 & ${ }^{6}$ (ii) & 8 & 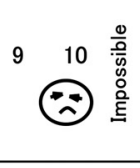 \\
\hline
\end{tabular}

Diagram showing page 2 of the English version of the Hand20 questionnaire.

explanatory illustrations which elderly people and children could easily understand. This illustrated questionnaire was named the Hand20 (Fig. 1).

Assessment of reliability, validity and responsiveness. All the study protocols were reviewed and approved by the
Institutional Review Board of Nagoya University. The subjects comprised 431 patients (192 men, 239 women) with disorders of the upper limb. They were asked to enrol in the study when their symptoms had been stable for the previous four weeks. Table I gives the diseases and injuries 
Table I. Diseases and injuries in participants in the study by number

\begin{tabular}{lc}
\hline Diseases and injuries & Number \\
\hline Entrapment neuropathy & 49 \\
Stenosing tenosynovitis & 41 \\
Peripheral nerve injury & 32 \\
Distal radial fracture & 30 \\
Phalangeal or metacarpal fracture & 30 \\
Osteoarthritis in the hand & 31 \\
Joint contracture & 19 \\
Carpal bone fracture or dislocation & 17 \\
Benign tumour & 16 \\
Elbow fracture or dislocation & 14 \\
Extensor tendon injury & 14 \\
Rheumatoid arthritis & 12 \\
Kienböck's disease & 10 \\
Finger amputation & 10 \\
Flexor tendon injury & 9 \\
Triangular fibrocartilage complex injury & 9 \\
Ganglion & 62 \\
Miscellaneous & 431 \\
Total &
\end{tabular}

included in our study and their frequency. The median age of the patients was 52 years (15 to 83 ). After informed consent had been obtained they completed both the Hand20 and DASH-JSSH questionnaires twice, with an interval of about ten days.

Reliability was investigated by examining the reproducibility and internal consistency based on the test-retest method. In order to determine validity, both construct and concurrent validity were measured. Construct validity reflects the ability of an instrument to measure an abstract concept or construct. Concurrent validity is a type of criterion-related validity and is demonstrated when a test correlates well with a simultaneously administered measure which has previously been validated. Criterion-based validity refers to the ability of a measurement to provide useful information in a specific situation. The completeness of item responses for the Hand20 was examined. A correlation coefficient between the Hand20 and DASH-JSSH was obtained. Concurrent validity was assessed on the basis that the Hand20 would have a strong correlation with the DASHJSSH and criterion-based validity in the belief that the Hand 20 would have a low correlation with age or gender.

Of the 431 patients with disorders of the upper limb, 157 (72 men, 85 women) who were to have surgical treatment were considered for inclusion in the examination of responsiveness. Their median age was 48 years (21 to 81 ). They were asked to complete both the Hand20 and DASH-JSSH questionnaires on two additional occasions, before and after surgery, to examine the effect size and the standardised response mean. Both are indices which measure the magnitude of a treatment effect.

Determination of norms for the Hand20. During the previous year, we had carried out a cross-sectional study to investigate the prevalence of arm, shoulder and hand pain among 2600 researchers and workers in the Faculty of Medicine and its affiliated hospital. We sent the Hand20 questionnaire, questions about their health status for the previous month and covering letters explaining our purpose and asking for participation in the study. Of the 1120 responders, 888 had no health problems. In this study, we used these data to analyse norms for the Hand20. Data were stratified according to gender, one of three age categories (18 to 29 years, 30 to 39 or 40 to 65 ), and vocational activities as classified by an international standard classification of occupations. ${ }^{20}$ Inclusion criteria were age between 18 and 65 years and absence of any health problems.

Statistical analysis. The interval measures (Hand20, DASH scores and age) were not normally distributed and therefore Spearman's correlation was used to find correlations and the Mann-Whitney $U$ test to make comparisons between genders. The number of patients with more than $10 \%$ of unanswered items in each of the two questionnaires was compared using the McNemar test. Cronbach's alpha was used to assess internal consistency. Instrument test-retest reliability was assessed using the intraclass correlation coefficient and construct validity and unidimensionality of the Hand 20 by using principal component analysis. The effect size was calculated by dividing the mean change in scores by the SD of initial scores and the standardised response mean by dividing the mean change in scores by the SD of that change. All the statistical analyses were carried out using SPSS version 17.0J software (SPSS Japan, Tokyo, Japan). The level of significance was set at $\mathrm{p} \leq 0.05$.

\section{Results}

Completeness of item responses. Most patients considered all the items of the Hand20 to be clear. The median and range of both the Hand20 and DASH-JSSH scores are shown in Table II. Cases which had more than $10 \%$ of the items incomplete were defined as inappropriate according to the instructions of DASH. Of the 431 patients, six $(1.4 \%)$ did not answer three or more items of the Hand 20 and $46(10.7 \%)$ did not answer four or more items of the DASH-JSSH. The Hand20 had significantly fewer inappropriate cases than the DASH-JSSH (McNemar test, $\mathrm{p}<0.001)$. Both the former and the latter were completed by 68 patients aged $>65$ years, three $(4.4 \%)$ of whom were inappropriate for the Hand20 and 19 (27.9\%) for the DASH-JSSH. Statistical analysis clearly showed that the Hand20 had significantly better completion of items than the DASH-JSSH $(\mathrm{p}<0.001)$ in elderly people (Table III).

Regarding the DASH-JSSH, items with a high unanswered rate among all patients were, in decreasing order, item 21 ('sexual activities'; 114 (26.5\%) of 431 patients), item 19 ('recreational activities in which you move your arm freely, e.g., playing frisbee, badminton, etc.'; $44(10.2 \%)$ of 431$)$, item 18 ('recreational activities in which you take some force or impact through your arm, shoulder or hand, e.g., golf, hammering, tennis, etc.', $43(10.0 \%)$ of 431 and item 8 ('yard work', 31 (7.2\%) of 
Table II. The median and range of Hand $20^{*}$ and DASHJSSH scores ${ }^{\dagger}$

\begin{tabular}{lll}
\hline Instrument scale & Median & Range \\
\hline Hand20 $(\mathrm{n}=425)$ & 28.0 & 0.0 to 98.0 \\
DASH-JSSH $(\mathrm{n}=385)$ & 22.5 & 0.0 to 89.2 \\
\hline
\end{tabular}

* Hand20 score, sum of $\mathrm{n}$ responses $\mathrm{n} \times 10$; DASH score (sum of $\mathrm{n}$ responses $/ \mathrm{n}$ ) $\times 25 ; \mathrm{n}$, number of completed responses

† DASH-JSSH, Japanese version of the Disabilities of the Arm, Shoulder and Hand (DASH)
Table III. Details of inappropriate cases for the Hand20 and DASH$\mathrm{JSSH}^{*}$

\begin{tabular}{|c|c|c|}
\hline \multirow[b]{2}{*}{ Instrument scale } & \multicolumn{2}{|l|}{ Numbers (\%) } \\
\hline & Total $(n=431)$ & Over 65 years of age $(n=68)$ \\
\hline Hand20 & $6(1.4)$ & $3(4.4)^{\dagger}$ \\
\hline DASH-JSSH & $46(10.7)$ & $19(27.9)$ \\
\hline
\end{tabular}

Table IV. Details of the standardised response mean (SRM) and effect size (ES) ( $n=157)$

\begin{tabular}{llllll}
\hline & Pre-operative & Post-operative & $\begin{array}{l}\text { Pre-post-operative } \\
\text { difference }\end{array}$ & \multicolumn{2}{l}{ Responsiveness } \\
\cline { 2 - 6 } Instrument scale & Mean (SD) & Mean (SD) & Mean (SD) & SRM & ES \\
\hline Hand20 & $36.5(28.2)$ & $21.3(24.9)$ & $-15.2(23.0)$ & -0.66 & -0.54 \\
DASH-JSSH $^{*}$ & $29.1(22.0)$ & $18.3(19.3)$ & $-10.8(16.1)$ & -0.68 & -0.49 \\
\hline
\end{tabular}

* DASH-JSSH, Japanese version of the Disabilities of the Arm, Shoulder and Hand (DASH)

431. Some generational and gender differences were noted in items with a high rate of missing data. Among men, item 4 ('prepare a meal', nine ( $4.7 \%$ ) of 192 patients) took third place and item 16 ('use a knife to cut food', eight $(4.2 \%)$ of 192 took fourth place. Items 18 and 19 took sixth place, with unanswered rates as low as five $(2.6 \%)$ of 192 patients. An unanswered rate of item 12 ('change a light bulb overhead') was also low (four $(2.1 \%)$ of 192 ). By contrast, among women, an unanswered rate of item 4 (five $(2.1 \%)$ of 239 patients) was quite low and item 16 took tenth place, with an unanswered rate as low as $14(5.9 \%)$ of 239 . Whereas item 19 took second place and item 18 took third place, with an unanswered rate as high as $39(16.3 \%)$ and $38(15.9 \%)$ of 239 patients respectively, item 12 took fourth place, with an unanswered rate as high as 20 $(8.4 \%)$ of 239 patients. In elderly people, item 19 took second place and item 18 third place, with a significantly high unanswered rate of $19(27.9 \%)$ and $17(25.0 \%)$ of 68 patients respectively. Item 16 took fifth place, with unanswered rate of ten $(14.7 \%)$ of 68 patients.

Reliability. The Cronbach's alpha coefficient for the 20 items in the Hand20 was high (0.973). When the alpha coefficient was calculated for each of the 20 items by eliminating each item one by one, the range was 0.971 to 0.974 , and no item was found to change the internal consistency substantially. The alpha coefficient for the 30 items in the DASH-JSSH was also high (0.973).

Instrument test-retest reliability was assessed using the intraclass correlation coefficient. The subject population for determining test-retest reliability was 431 patients, and the median period between the first and second tests was seven days (2 to 57). Intraclass correlation coefficients for the Hand20 and DASH-JSSH were 0.943 (95\% confidence interval (CI) 0.931 to 0.952 ) and 0.930 (95\% CI 0.915 to
0.943), respectively. The intraclass correlation coefficient for the Hand20 indicated sufficient reproducibility.

Validity. Principal component analysis was performed to confirm the unidimensionality of the Hand20. The first factor of the Hand20 had an eigenvalue (amount of variation in the total sample accounted for by that factor) of 13.4, explaining $66.9 \%$ of the total variance in the Hand20 scores. Unidimensionality was found to be strong as a result of a substantial difference between the first and second factors (eigenvalue 1.05). When examining the first factor loading for each item, all items had a loading (correlation with total score) of $\geq 0.6$.

The correlation coefficient between the Hand20 and DASH-JSSH was $0.91(p<0.001)$, indicating a strong correlation. This result supports the hypotheses set down previously relating to concurrent validity.

Regarding criterion-based validity, Hand20 scores for men (median 28.0 (0.0 to 98.0) and women (median 31.0, 0.0 to 90.5 ) were compared using the Mann-Whitney U test, and no significant differences were seen between genders $(\mathrm{p}=0.315)$. The correlation coefficient between the Hand20 score and age was $r=0.119(p=0.014)$.

Responsiveness. Cohen classified $<0.5$ as a small effect, 0.5 to 0.8 as medium and $>0.8$ as large. ${ }^{21}$ The median period between before and after surgical treatment was 117 days (9 to 184). The standardised response mean for the Hand 20 and DASH-JSSH was -0.66 and -0.68 , respectively and the effect size -0.54 and -0.49 , respectively (Table IV).

Norms of the Hand20. A total of 1120 employees completed the Hand20 questionnaire. The data of 888 employees who had no health problems were used to obtain the norms. The median age was 32 years (21 to 65). The median Hand20 score was 0 (0 to 66). When data 
were stratified according to age, the median Hand20 scores was 0 ( 0 to 20$)$ for age 18 to 29 years, 0 ( 0 to 66 ) for age 30 to 39 and 0 ( 0 to 24 ) for age 40 to 65 .

\section{Discussion}

The DASH questionnaire is one of the best and most widely used instruments for evaluating patients with disorders involving multiple joints in the upper limb. ${ }^{22}$ The DASH and Quick DASH questionnaire are recommended for patients between 18 and 65 years of age. ${ }^{18}$ However, with an ageing society, the development of instruments to assess the health status of older people has become an important issue and our study showed that almost onethird of patients over 65 years of age could not complete the DASH properly. In order to expand the age range we developed the Hand20. We took the lower ability for reading comprehension of elderly individuals into consideration and tried to use short, clear questions which were minimally affected by cultural differences, with the expectation of significant improvement in the understandability as reported by others. ${ }^{23}$ However, Walmsley, Scott and Lehrer ${ }^{24}$ have suggested that the readability level of a document is a poor indicator of comprehension by elderly subjects and that simplifying the language is not enough to aid comprehension. We therefore added explanatory illustrations. These have been found to improve the comprehension of medication labels or health-education leaflets, if the illustrations and text are well matched. ${ }^{25,26}$ Patients with low literacy particularly benefit from illustrations. ${ }^{26}$

The development of the Hand20 questionnaire followed a systematic standardised approach. ${ }^{27}$ As for internal consistency, Cronbach's alpha coefficient for the Hand20 (0.97) was equivalent to that for the DASH-JSSH (0.97). Internal consistency needs to be $>0.90$ if a scale is to be used for tracking individual patients. Thus, the Hand20 should be used with caution for assessment of individual patients over time. As for reproducibility, the intraclass correlation coefficient of the Hand20 (0.94) was equivalent to that of the DASH-JSSH (0.93). The results for internal consistency and reproducibility of the Hand20 indicated sufficient reliability. Many types of validation are available for health-status measures. We used the correlation coefficient between the Hand20 and DASH-JSSH, which was 0.91. This strong correlation supported the validity of the Hand20. Another method of validation is principal component analysis. The Hand20 scale had high unidimensionality, with no low item-scale correlation. These results were similar to those for the DASH-JSSH. ${ }^{9}$ Loading of this scale was very high. Our results showed that the Hand20 scale had high validation. Cohen's rule-of-thumb for interpreting the 'effect size index' can be applied to the standardised response mean. ${ }^{28}$ The responsiveness of the Hand20 and DASHJSSH scales for patients with disorders of the upper limb was moderate.
In our study we tried to establish population-based normative data for the Hand20. Comparisons of the normative data allow research workers to assess whether the physical status of the patients who have been treated is abnormal and whether it has returned to normal after treatment. In this context, the lower the level of norm as well as the lower the level of variation which an instrument has, the more advantageous it is for assessment. To the best of our knowledge, two groups have studied the norms of the DASH independently, one in the United States and the other in Germany. They have reported the means of the norms of the DASH score to be 10.10 (SD 14.78) and 13.0 (SD $15.0),{ }^{29,30}$ respectively. Unfortunately, because of the skew in the data, we reported the norms of the Hand20 score using the median. Therefore, we were unable to compare our results with those of the two studies. Quoting means and SDs when the data are clearly skewed is not appropriate, but the mean of the norms of the Hand20 score was 1.2 (SD 3.9). This suggested that the norm of the Hand20 was much smaller than that of the DASH.

One of the present authors $(\mathrm{HH})$ was involved in the development of the Japanese version of the DASH questionnaire. ${ }^{9}$ In the cross-cultural translation and adaptation, he encountered a number of problems. According to the guidelines for cross-cultural translation of the DASH published by the American Academy of Orthopaedic Surgeons (AAOS), there are several possible problems. The most difficult is adaptation for use either in another country or in another language. ${ }^{17}$ Therefore, the fact that more than $10 \%$ of patients left more than three items unanswered and were judged to be inappropriate for evaluation in our study is not surprising. However, detailed analysis of the unanswered questions suggested that patients did not answer some items not because of cultural differences, but because of lack of experience or knowledge. In fact, items 18 and 19 were listed as frequently missed items for both genders, although there was a significant difference in the unanswered rate between the genders. A possible reason for this discrepancy is that, in Japan, women are much less interested in sports than men. Another example is item 12, which was listed as a frequently unanswered item only for women. In Japan, most people do not use light bulbs. Instead they use fluorescent lamps in the house and lighting equipment is usually fixed by men. Alotaibi ${ }^{31}$ carried out a comprehensive review of articles regarding the cross-cultural translation of the DASH and found that particular items were more difficult to translate than others because of a vagueness in explanation (item 8 'yard work' and item 20 'manage transportation needs'), lack of knowledge or understanding (item 17 'recreational activities which require little effort, e.g., card playing, knitting, etc'), and cultural norms (item 4 'prepare a meal' and item 21 'sexual activities'). In addition, activities given as examples in item 18 'recreational activities in which you take some force or impact through your arm, shoulder, or hand (e.g. golf, hammering, tennis, etc)' and item 19 'recreational activities in which you move your arm freely (e.g. playing 
frisbee, badminton, etc)' were unknown, infrequently used, less applicable and/or inappropriate even to people of Western European countries including Germany, Sweden and Greece. ${ }^{11,12,15,32,33}$ Both our study and that by Alotaibi ${ }^{31}$ clearly showed that cultural differences may not represent the biggest obstacle for cross-cultural adaptation of the DASH, and that many issues can be resolved by careful selection of items and the use of clear explanations.

Although some elderly individuals maintain high intellectual activity, deterioration of comprehension is part of ageing. It is also true that there are considerable generational differences with respect to cultural and social norms. The incidence of inappropriate answers for the DASH questionnaire was as high as $28 \%$ among elderly patients in our study. Significantly better completeness of item responses for the Hand20 supported our hypothesis that the combined use of simplified language and explanatory illustrations improved comprehension in elderly subjects. We therefore believe that the Hand20 questionnaire may be used to evaluate patients with disorders of the upper limb regardless of their intellectual level.

Regarding the English version of the Hand20 questionnaire, we carefully translated a Japanese version to create an equivalent version in English. First, a translation was made by a bilingual translator whose mother tongue was Japanese. This was then corrected by an independent bilingual translator whose mother tongue was English. Then, the English version was translated back into Japanese by another independent translator to check for inconsistency or ambiguity. Therefore we believe that the English version of the Hand20 questionnaire is linguistically appropriate. However, this may not be enough to confirm the equivalence of these questionnaires, because the questionnaire must also be adapted culturally. ${ }^{34}$ Therefore, we hope that many clinicians and research workers will find the Hand20 scale useful and introduce it into their countries after an appropriate validation process.

We would like to thank A. Morita, A. Okano, K. Nishikawa, M. Chaki, M. Yamamoto, S.- Koh and all other members of the Hand Frontier for their support with the development of the Hand20 and data collection.

No benefits in any form have been received or will be received from a commercial party related directly or indirectly to the subject of this article.

\section{References}

1. Dawson J, Carr A. Outcomes evaluation in orthopaedics. J Bone Joint Surg [Br] 2001;83-B:313-15.

2. Constant CR, Murley AH. A clinical method of functional assessment of the shoulder. Clin Orthop 1987;214:160-4.

3. King GJ, Richards RR, Zuckerman JD, et al. A standardized method for assessment of elbow function: Research Committee, American Shoulder and Elbow Surgeons. J Shoulder Elbow Surg 1999;8:351-4.

4. Kirkley A, Griffin S, McLintock H, Ng L. The development and evaluation of a disease-specific quality of life measurement tool for shoulder instability: The Western Ontario Shoulder Instability Index (WOSI). Am J Sports Med 1998;26:764-72.

5. Levine DW, Simmons BP, Koris MJ, et al. A self-administered questionnaire for the assessment of severity of symptoms and functional status in carpal tunnel syndrome. J Bone Joint Surg [Am] 1993;75-A:1585-92.

6. Hudak PL, Amadio PC, Bombardier C. Development of an upper extremity outcome measure: the DASH (disabilities of the arm, shoulder and hand [corrected]: The Upper Extremity Collaborative Group (UECG). Am J Ind Med 1996;29:602-8.

7. Beaton DE, Katz JN, Fossel AH, et al. Measuring the whole or the parts?: validity, reliability, and responsiveness of the Disabilities of the Arm, Shoulder and Hand out come measure in different regions of the upper extremity. J Hand Ther 2001;14:128-46.
8. MacDermid JC, Richards RS, Donner A, Bellamy N, Roth JH. Responsiveness of the short form-36, disability of the arm, shoulder, and hand questionnaire, patientrated wrist evaluation, and physical impairment measurements in evaluating recovery after a distal radius fracture. J Hand Surg Am 2000;25:330-40.

9. Imaeda T, Toh S, Nakao Y, et al. Validation of the Japanese Society for Surgery of the Hand version of the Disability of the Arm, Shoulder, and hand questionnaire. $J$ Orthop Sci 2005; 10:353-9.

10. Imaeda T, Hirata H, Toh S, et al. Comparative responsiveness of Japanese versions of the DASH and SF-36 questionnaires and physical measurement to clinical changes after carpal tunnel release. Hand Surg 2006;11:27-33.

11. Atroshi I, Gummesson C, Andersson B, Dahlgren E, Johansson A. The disabilities of the arm, shoulder and hand (DASH) outcome questionnaire: reliability and validity of the Swedish version evaluated in 176 patients. Acta Orthop Scand 2000;71:613-18.

12. Offenbäacher M, Ewert T, Sangha 0, Stucki G. Validation of a German version of the Disabilities of Arm, Shoulder, and Hand questionnaire (DASH-G). Z Rheumatol 2003;62:168-77

13. Rosales RS, Delgado EB, Díez de la Lastra-Bosch I. Evaluation of the Spanish version of the DASH and carpal tunnel syndrome health-related quality-of-life instruments: cross-cultural adaptation process and reliability. I Hand Surg Am 2002;27:334-43

14. Padua R, Padua L, Ceccarelli E, et al. Italian version of the Disability of the Arm, Shoulder and Hand (DASH) questionnaire: cross-cultural adaptation and validation. $J$ Hand Surg Br 2003;28:179-86

15. Lee EW, Lau JS, Chung MM, Li AP, Lo SK. Evaluation of the Chinese version of the Disability of the Arm, Shoulder and Hand (DASH-HKPWH): cross-cultural adaptation process, internal consistency and reliability study. J Hand Ther 2004;17:417-23.

16. Lee EW, Chung MM, Li AP, Lo SK. Construct validity of the Chinese version of the disabilities of the arm, shoulder and hand questionnaire (DASH-HKPWH). J Hand Surg Br 2005;30:29-34.

17. Beaton DE, Bombardier C, Guillemin F, Ferraz MB. Guidelines for the process of cross-cultural adaptation of self-report measures. Spine 2000;25:3186-91

18. No authors listed. The DASH outcome measure: frequently asked questions. http:/ /www.dash.iwh.on.ca/faq.htm (date last accessed 9 March 2010).

19. Hayes V, Morris J, Wolfe C, Morgan M. The SF-36 health survey questionnaire: is it suitable for use with older adults? Age Ageing 1995;24:120-5

20. No authors listed. International Standard Classification of Occupations. International Labour Organization: ISCO-88. http://www.ilo.org/public/english/bureau/stat/ isco/isco88/index.htm (date last accessed 9 March 2010).

21. Cohen J. Statistical power analysis for the behavioral sciences. Second edition. Hillsdale: Lawrence Erlbaum Associates, 1988:147-51.

22. Changulani M, Okonkwo U, Keswani T, Kalairajah Y. Outcome evaluation measures for wrist and hand: which one to choose? Int Orthop 2008;32:1-6.

23. Boulos MNK. Readability assessment of british internet information resources on diabetes mellitus targeting lay persons. University of Bath, 2004. http://healthcybermap.semanticweb.org/publications/readability.ppt (date last accessed 9 March 2010).

24. Walmsley SA, Scott K, Lehrer R. Effects of document simplification on the reading comprehension of the elderly. Journal of Reading Behavior 1981;13:237-48.

25. Patel VL, Branch T, Arocha JF. Errors in interpreting quantities as procedures: the case of pharmaceutical labels. Int J Med Inform 2002;65:193-211.

26. Houts PS, Doak CC, Doak LG, Losclazo MJ. The role of pictures in improving health communication: a review of research on attention, comprehension, recall, and adherence. Patient Educ Couns 2006;61:173-90.

27. Guyatt GH, Bombardier C, Tugwell PX. Measuring disease-specific quality of life in clinical trials. CMAJ 1986;134:889-95.

28. Liang MH, Fossel AH, Larson MG. Comparison of five health status instruments for orthopedic evaluation. Med Care 1990;28:632-42.

29. Hunsaker FG, Cioffi DA, Amadio PC, Wright JG, Caughlin B. The American academy of orthopaedic surgeons outcomes instruments: normative values from the general population. J Bone Joint Surg [Am] 2002;84-A:208-15.

30. Jester A, Harth A, German G. Meauring levels of upper-extremity disability in employed adults using the DASH questionnaire. J Hand Surg Am 2005;30:1074.

31. Alotaibi NM. The cross-cultural adaptation of the disability of arm, soulder and hand (DASH): a systematic review. Occup Ther Int 2008;15:178-90.

32. Orfale AG, Araújo PM, Ferrax MB, Natour J. Translation into Brazilian Portuguese, cultural adaptation and evaluation of the reliability of the Disabilities of the Arm, Shoulder and Hand Questionnaire. Braz J Med Biol Res 2005;38:293-302.

33. Themistocleous GD, Goudelis G, Kyrou I, et al. Translation into Greek, cross-cultural adaptation and validation of the Disabilities of the Arm, Shoulder, and Hand Questionnaire (DASH). J Hand Ther 2006;19:350-7.

34. Guillemin F, Bombardier C, Beaton D. Cross-cultural adaptation of health-related quality of life measures: literature review and proposed guidelines. J Clin Epidemiol 1993;46:1417-32. 